\title{
Оптические свойства полиэтилена, наполненного нанокристаллитами $\mathrm{Bi}_{2} \mathrm{Te}_{3}$
}

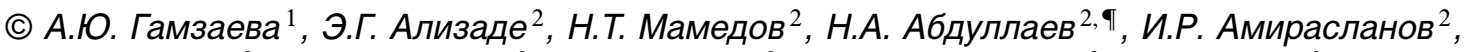 \\ Е.Н. Алиева ${ }^{2}$, Х.Н. Ахмедова ${ }^{2}$, Г.Х. Аждаров ${ }^{2}$, К.Ш. Кахраманов ${ }^{2}$, С.А. Немов ${ }^{3}$
}

${ }^{1}$ Гянджинский государственный университет,

Az-2003 Гянджа, Азербайджан

${ }^{2}$ Институт фризики Национальной академии наук Азербайджана,

Az-1143 Баку, Азербайджан

${ }^{3}$ Санкт-Петербургский государственный политехнический университет Петра Великого, 195251 Санкт-Петербург, Россия

『 E-mail: abnadir@mail.ru

(Получена 21 мая 2018 г. Принята к печати 10 сентября 2018 г.)

\begin{abstract}
Путем термического прессования полиэтилена низкой плотности и $\mathrm{Bi}_{2} \mathrm{Te}_{3}$ в порошкообразном состоянии с размерами нанокристаллов $\sim 50$ нм, получены композитные смеси с различной концентрацией составляющих. Полученные образцы, предварительно охарактеризованные методами рентгеновской дифрактометрии и комбинационного рассеяния света, исследованы методом спектроскопической эллипсометрии в интервале энергий фотонов 1-6эВ. На основе измеренных оптических констант полиэтилена и кристалла $\mathrm{Bi}_{2} \mathrm{Te}_{3}$, в приближении эффективной среды Бруггемана, рассчитаны диэлектрические функции для композитов с номинальной весовой концентрацией $\mathrm{Bi}_{2} \mathrm{Te}_{3} 5,10,30,40,50,60$ и 70\%. Анализ деполяризационных особенностей отраженного света позволил установить реальную неоднородность исследуемых композитных образцов, обусловленную кластеризацией нанокристаллитов при увеличении весовой концентрации $\mathrm{Bi}_{2} \mathrm{Te}_{3}$ в полиэтилене и предложить наиболее достоверную оптическую модель полученных композитов.
\end{abstract}

DOI: 10.21883/FTP.2019.02.47105.8912

\section{1. Введение}

Как известно, соединения типа $\mathrm{Bi}_{2} \mathrm{Te}_{3}$ и твердые растворы на их основе нашли практическое применение в качестве термоэлектрических материалов. Для более широкого использования необходимо повышение термоэлектрической эффективности, что обычно достигается легированием этих соединений или подбором определенных составов твердых растворов. Теоретические расчеты [1] указывают, что значительного увеличения термоэлектрической эффективности можно достичь использованием низкоразмерных структур (тонкие пленки, нанонити, нанокристаллы и т.п.) на основе этих соединений.

Нанокомпозиты были получены путем термического прессования полиэтилена низкой плотности (LDPE Low Density Polyethylene) и $\mathrm{Bi}_{2} \mathrm{Te}_{3}$ в порошкообразном состоянии с массовой концентрацией $5,10,20,30,40$, 50, 60 и 70\% [2]. Предварительно порошок просеивался через специальное ситечко и размеры нанокристаллитов $\mathrm{Bi}_{2} \mathrm{Te}_{3}$ не превышали 50 нм.

Цель настоящей статьи заключается в исследовании оптических свойств нанокомпозитов - матрицы LDPE с наполнителем в виде нанокристаллитов $\mathrm{Bi}_{2} \mathrm{Te}_{3}$.

\section{2. Характеризация нанокомпозитов}

Характеризация нанокомпозитных образцов проводилась методами дифракции рентгеновских лучей и комбинационного рассеяния света.
Рентгенодифрактометрические исследования проводились на рентгеновском дифрактометре Bruker D2PHASER. На рис. 1 представлены дифрактограммы нанокомпозитов LDPE- $\mathrm{Bi}_{2} \mathrm{Te}_{3}$ различной концентрации $(5,10,30,40,50,60$ и 70\%). Хорошо заметно, что при низких концентрациях $\mathrm{Bi}_{2} \mathrm{Te}_{3}(5-10 \%)$ при углах $2 \theta$ примерно равными 21.6 и $24.0^{\circ}$, по значению

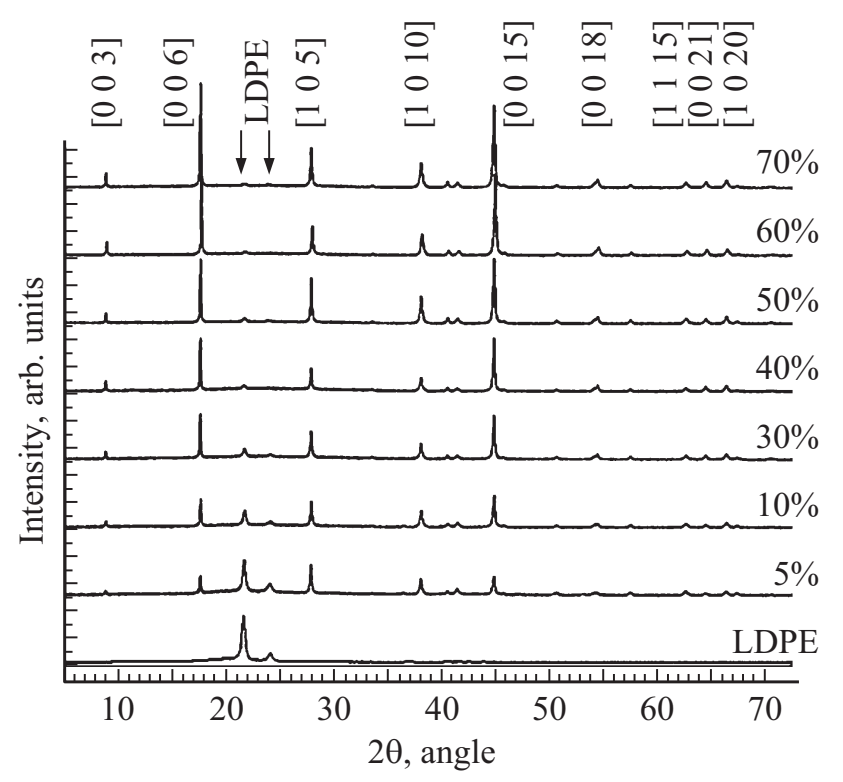

Рис. 1. Дифракция рентгеновских лучей в нанокомпозитах $\mathrm{LDPE}-\mathrm{Bi}_{2} \mathrm{Te}_{3}$ с различной концентрацией наночастиц $(5,10$, $30,40,50,60$ и $70 \%)$. 
Таблица 1. Средние размеры кристаллитов $\mathrm{Bi}_{2} \mathrm{Te}_{3}$ в нанокомпозитах

\begin{tabular}{c|c|c}
\hline $\begin{array}{c}\text { Содержание } \\
\mathrm{Bi}_{2} \mathrm{Te}_{3}, \%\end{array}$ & $\begin{array}{c}\text { Полуширина } \\
\text { спектральной линии, град }\end{array}$ & $\begin{array}{c}\text { Размеры } \\
\text { кристаллитов, нм }\end{array}$ \\
\hline 5 & 0.245 & 33.2 \\
10 & 0.255 & 31.9 \\
30 & 0.252 & 32.3 \\
40 & 0.239 & 34.0 \\
50 & 0.278 & 29.3 \\
60 & 0.291 & 27.9 \\
70 & 0.299 & 27.2
\end{tabular}

интенсивностей доминируют рефлексы, характерные для матрицы - LDPE. C увеличением концентрации $\mathrm{Bi}_{2} \mathrm{Te}_{3}$ интенсивность рефлексов, характерных для $\mathrm{Bi}_{2} \mathrm{Te}_{3}$, возрастает, а интенсивность рефлексов при углах 21.6 и $24^{\circ}$ существенно падает.

В табл. 1 приведены средние размеры нанокристаллитов, оцененные по формуле Шеррера. Как видно из табл. 1, средние размеры нанокристаллитов действительно находятся в пределах 50 нм и уменьшаются на $\sim 20 \%$ с увеличением концентрации $\mathrm{Bi}_{2} \mathrm{Te}_{3}$. Однако последняя тенденция вряд ли свидетельствует о реальных изменениях размеров нанокристаллитов ввиду больших погрешностей при их оценке, обусловленных слабой интенсивностью дифракционных пиков при низкой концентрации $\mathrm{Bi}_{2} \mathrm{Te}_{3}$.

Исследования комбинационного рассеяния (КР) света проводились на трехмерном конфокальном рамановском микроспектрометре Nanofinder 30 (Tokyo Instr.), длина волны возбуждения $\lambda=532$ нм. Радиус сечения

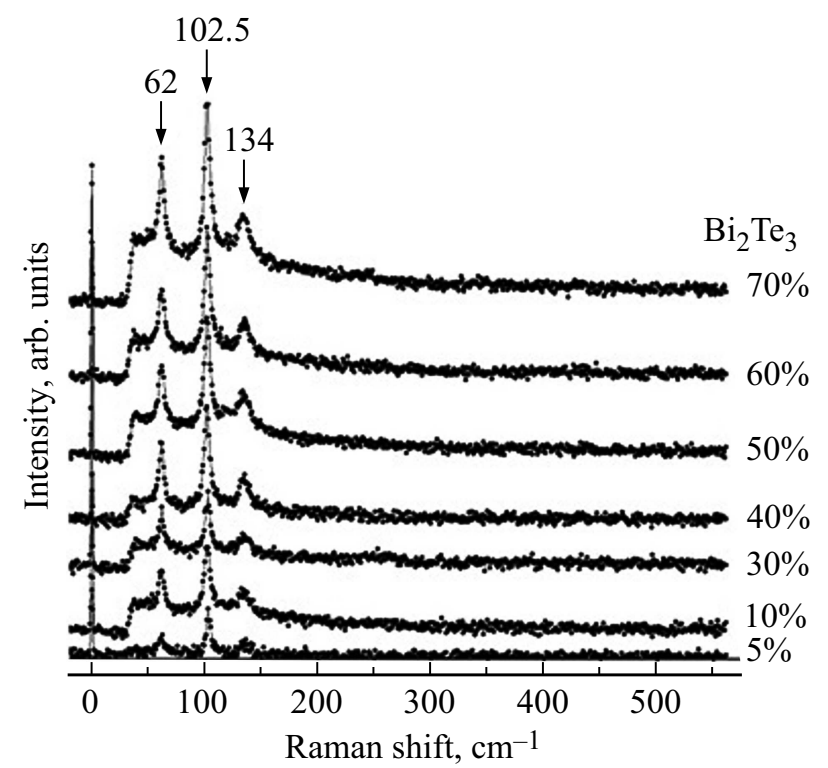

Рис. 2. Спектры комбинационного рассеяния нанокомпозитов с различными концентрациями $\mathrm{Bi}_{2} \mathrm{Te}_{3}(5,10,30,40,50,60$ и $70 \%)$. падающего на нанокомпозит лазерного луча был равен $\sim 4$ мкм. Исследования проводились в геометрии обратного рассеяния. Приемником излучения служила охлаждаемая $\mathrm{CCD}$-камера $\left(-70^{\circ} \mathrm{C}\right)$, работающая в режиме счета фотонов. Все измерения сделаны при времени экспозиции 20 с и мощности возбуждающего излучения $10 \mathrm{MBT}$. Точность определения положения спектральной линии была не хуже $0.5 \mathrm{~cm}^{-1}$. Результаты исследований спектров комбинационного рассеяния на нанокомпозитах с различными концентрациями наполнителя $\mathrm{Bi}_{2} \mathrm{Te}_{3}(5,10,30,40,50,60$ и 70\%) представлены на рис. 2. Хорошо видно, что в данной геометрии рассеяния для всех концентраций наполнителя $\mathrm{Bi}_{2} \mathrm{Te}_{3}$ отчетливо наблюдаются характерные для $\mathrm{Bi}_{2} \mathrm{Te}_{3}$ три КР-активные моды [3]: на частоте $62 \mathrm{~cm}^{-1}\left(A_{1 g}^{1}\right)$, на частоте $102.5 \mathrm{~cm}^{-1}\left(E_{g}^{2}\right)$ и на частоте $134 \mathrm{~cm}^{-1}\left(A_{1 g}^{2}\right)$. С увеличением концентрации наполнителя $\mathrm{Bi}_{2} \mathrm{Te}_{3}$ интенсивность рамановских мод возрастает.

\section{3. Эллипсометрические измерения и анализ полученных данных}

Для исследования оптических параметров нанокомпозитов нами были проведены спектральные эллипсометрические исследования, являющиеся высокочувствительным и точным оптическим методом исследования поверхностей и границ раздела различных сред. Этот метод основан на изучении изменения состояния поляризации отраженного света после взаимодействия его с поверхностью границ раздела этих сред. Измерения проводились на эллипсометре оптического диапазона M-2000 DI (J.A. Woollam Co, Inc.). Спектральная зависимость эллипсометрических параметров $\Delta$ и $\Psi$ снималась в диапазоне энергий фотонов 1-6эВ с шагом 50 мэВ при углах падения в диапазоне $60-75^{\circ}$.

Основное уравнение эллипсометрии, связывающее между собой эллипсометрические параметры $\Delta, \Psi$ и комплексные значения коэффициентов отражения Френеля $r_{p}$ и $r_{s}$ для $p$ и $s$ компонентов эллиптически поляризованного света, записывается в виде [4]

$$
\operatorname{tg}(\Psi) \exp (i \Delta)=\frac{r_{p}}{r_{s}}
$$

Выбор адекватной оптической модели, правильно описывающей отражающие свойства исследуемого образца, является одним из важных этапов при эллипсометрических исследованиях. В наших исследованиях наиболее подходящей оптической моделью оказалась система, содержащая один и более слоев с разной стехиометрией, так как в образцах отсутствовала единая стехиометрия из-за кластеризации наночастиц и полиэтилена. Таким образом, для решения обратной эллипсометрической задачи в связи с неоднородностью нанокомпозитов нами была выбрана модель „однородная подложка - однородный слой“ [4]. Вычисления проводились на основе 

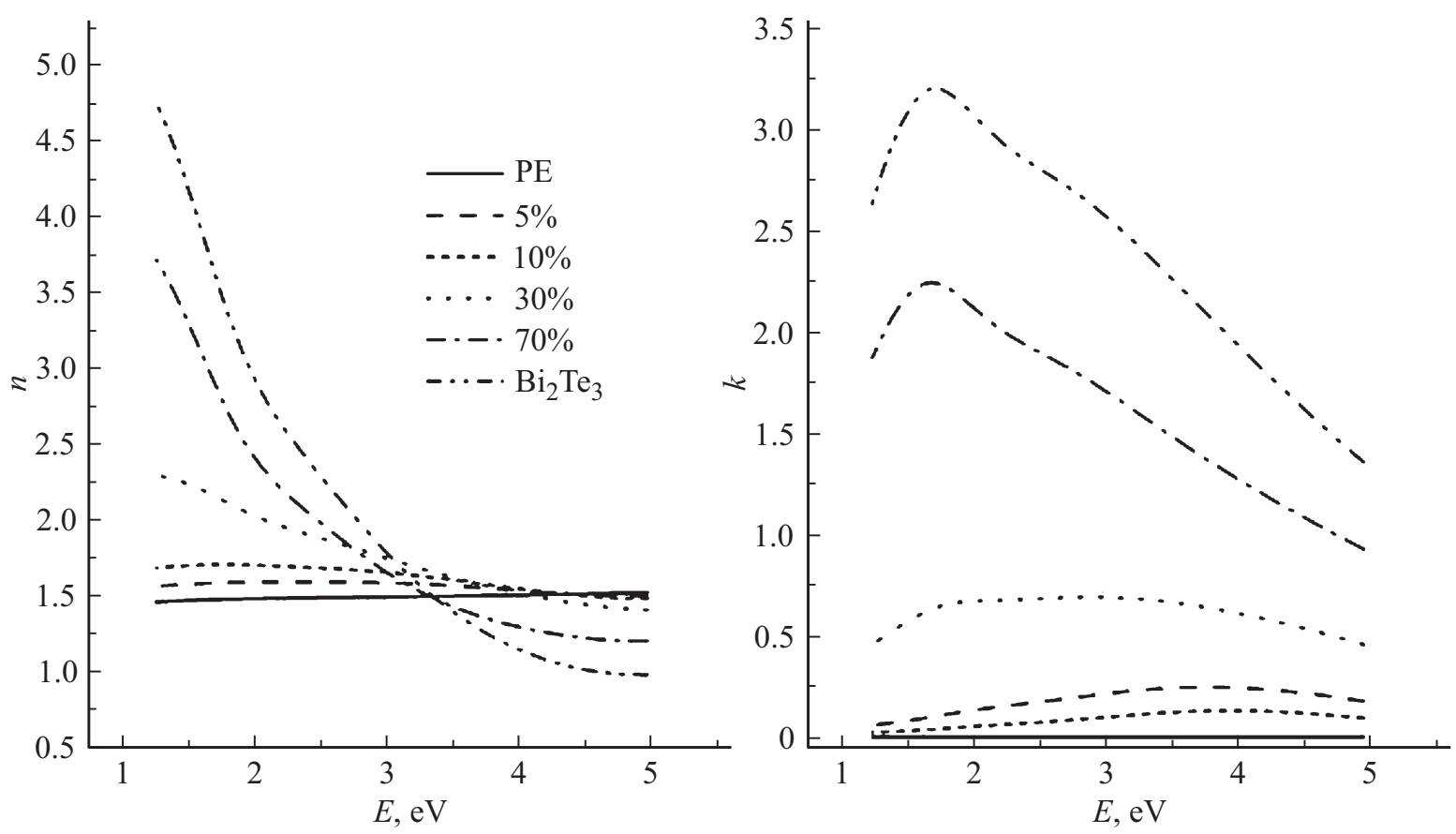

Рис. 3. Показатели преломления и экстинкции, рассчитанные при указанных номинальных концентрациях, в диапазоне энергий $1-5$ эВ.

аппроксимации эффективной среды (Effective Medium Approximation, EMA) приближением Бруггемана [5]:

$$
\sum_{j=1}^{n} \rho_{j} \frac{\varepsilon_{j}-\varepsilon_{e f}}{\varepsilon_{j}+2 \varepsilon_{e f}}=0
$$

Здесь $\rho_{j}-$ доля $j$-го вещества, $\varepsilon_{j}-$ диэлектрическая постоянная $j$-го вещества, $\varepsilon_{e f}$ - эффективная диэлектрическая постоянная.

Так как $\varepsilon-$ комплексная величина, для него запишем

$$
\varepsilon=\varepsilon_{r}+i \varepsilon_{i} .
$$

Для расчета $n$ и $k$, показателя преломления и экстинкции соответственно применялись формулы (4) и (5):

$$
\begin{gathered}
n=\sqrt{\frac{\varepsilon_{r}+\sqrt{\varepsilon_{r}^{2}+\varepsilon_{i}^{2}}}{2}}, \\
k=\frac{\varepsilon_{i}}{\sqrt{2\left(\varepsilon_{r}+\sqrt{\varepsilon_{r}^{2}+\varepsilon_{i}^{2}}\right)}} .
\end{gathered}
$$

Приближение Бруггемана для слоев нанокомпозитов, состоящих из двух компонентов - наночастиц $\mathrm{Bi}_{2} \mathrm{Te}_{3}$ и полиэтилена, примет вид

$$
\rho_{\mathrm{Bi}_{2} \mathrm{Te}_{3}} \frac{\varepsilon_{\mathrm{Bi}_{2} \mathrm{Te}_{3}}-\varepsilon_{e f}}{\varepsilon_{\mathrm{Bi}_{2} \mathrm{Te}_{3}}+2 \varepsilon_{e f}}+\rho_{\mathrm{PE}} \frac{\varepsilon_{\mathrm{PE}}-\varepsilon_{e f}}{\varepsilon_{\mathrm{PE}}+2 \varepsilon_{e f}}=0 .
$$

Здесь $\varepsilon_{\mathrm{Bi}_{2} \mathrm{Te}_{3}}$ и $\rho_{\mathrm{PE}}-\mathrm{Bi}_{2} \mathrm{Te}_{3}$ и $\mathrm{PE}$ соответственно, $\varepsilon_{\mathrm{Bi}_{2} \mathrm{Te}_{3}}$ - диэлектрическая постоянная $\mathrm{Bi}_{2} \mathrm{Te}_{3}, \varepsilon_{\mathrm{PE}}-$ диэлектрическая постоянная $\mathrm{PE}, \varepsilon_{e f}-$ эффективная диэлектрическая постоянная.

Применив приближение Бруггемана (6), для указанных номинальных концентраций были рассчитаны спектральные зависимости показателя преломления и коэффициента экстинкции, представленные на рис. 3 . Из рис. 3 становится понятным, какой должна быть дисперсия в идеальном нанокомпозите с увеличением содержания наночастиц $\mathrm{Bi}_{2} \mathrm{Te}_{3}$.

Однако наблюдается отклонение расчетных величин показателей преломления и экстинкции (рис. 3 ) от измеренных экспериментально, что указывает на неоднородность образцов. При указанной технологии получения неизбежно внедрение в нанокомпозиты воздушных полостей. Для слоев, содержащих еще и третий компонент „воздух“ (void), приближение Бруггемана примет вид

$$
\begin{aligned}
\rho_{\mathrm{Bi}_{2} \mathrm{Te}_{3}} & \frac{\varepsilon_{\mathrm{Bi}_{2} \mathrm{Te}_{3}}-\varepsilon_{e f}}{\varepsilon_{\mathrm{Bi}_{2} \mathrm{Te}_{3}}+2 \varepsilon_{e f}}+\rho_{\mathrm{PE}} \frac{\varepsilon_{\mathrm{PE}}-\varepsilon_{e f}}{\varepsilon_{\mathrm{PE}}+2 \varepsilon_{e f}} \\
& +\left(1-\rho_{\mathrm{Bi}_{2} \mathrm{Te}_{3}}-\rho_{\mathrm{PE}}\right) \frac{\varepsilon_{\text {Void }}-\varepsilon_{e f}}{\varepsilon_{\text {Void }}+2 \varepsilon_{e f}}=0 .
\end{aligned}
$$

Здесь $\varepsilon_{\text {Void }}$ - диэлектрическая постоянная воздуха.

Необходимо отметить, что физически разумная модель с наименьшей средней стандартной ошибкой (MSE, Mean Standard Error) считается наиболее достоверной. Нами были вычислены величины MSE для всех концентраций нанокомпозитов. Оказалось, что для нанокомпозитов с содержанием наночастиц $\mathrm{Bi}_{2} \mathrm{Te}_{3}$ концентрациями $5,10,30,40$ и $50 \%$ является подходящей модель „один слой-подложка“. Однако для концентраций 60 и $70 \%$ 
Таблица 2. Величины MSE для нанокомпозитов с различной номинальной концентрацией $\mathrm{Bi}_{2} \mathrm{Te}_{3}$

\begin{tabular}{c|c|c|c|c|c|c|c|c}
\hline $\begin{array}{c}\text { Концентрация } \\
\mathrm{Bi}_{2} \mathrm{Te}_{3}, \%\end{array}$ & 5 & 10 & 20 & 30 & 40 & 50 & 60 & 70 \\
\hline MSE & 5.7 & 6.7 & 7.1 & 5.3 & 6.6 & 7.8 & 5.1 & 6.9
\end{tabular}

Таблица 3. Концентрации и толщины слоев в рамках однослойной модели

\begin{tabular}{c|c|c|c|c|c|c|c}
\hline \multicolumn{2}{c|}{ Содержание $\mathrm{Bi}_{2} \mathrm{Te}_{3}, \%$} & 5 & 10 & 20 & 30 & 40 & 50 \\
\hline Подложка & Толщина, мкм & 200 & 200 & 200 & 200 & 200 & 200 \\
& $\mathrm{Bi}_{2} \mathrm{Te}_{3}, \%$ & 5 & 8.7 & 10.6 & 10.1 & 11.7 & 11.6 \\
& Воздух, \% & 1 & 16.1 & 26.5 & 27.8 & 31.1 & 10.3 \\
\hline \multirow{2}{*}{ Слой 1 } & Толщина, нм & 63 & 53 & 49 & 48 & 46 & 56 \\
& Вi $_{2} \mathrm{Te}_{3}, \%$ & 2.8 & 4.8 & 5.5 & 5.3 & 6 & 7.9
\end{tabular}

Таблица 4. Концентрации и толщины слоев в рамках трехслойной модели

\begin{tabular}{|c|c|c|c|}
\hline \multicolumn{2}{|c|}{ Концентрация $\mathrm{Bi}_{2} \mathrm{Te}_{3}, \%$} & 60 & 70 \\
\hline Подложка & $\begin{array}{c}\text { Толщина, мкм } \\
\mathrm{Bi}_{2} \mathrm{Te}_{3}, \% \\
\text { Воздух, \% }\end{array}$ & $\begin{array}{c}200 \\
23.4 \\
1\end{array}$ & $\begin{array}{l}200 \\
49.5 \\
16.1\end{array}$ \\
\hline Слой 1 & $\begin{array}{c}\text { Толщина, нм } \\
\mathrm{Bi}_{2} \mathrm{Te}_{3}, \% \\
\text { Воздух, \% }\end{array}$ & $\begin{array}{r}138 \\
39.3 \\
58.1\end{array}$ & $\begin{array}{l}108 \\
26.8 \\
58.7\end{array}$ \\
\hline Слой 2 & $\begin{array}{c}\text { Толщина, нм } \\
\mathrm{Bi}_{2} \mathrm{Te}_{3}, \% \\
\text { Воздух, \% }\end{array}$ & $\begin{array}{c}25 \\
16.7 \\
2.8\end{array}$ & $\begin{array}{c}50 \\
23.8 \\
2.5\end{array}$ \\
\hline Слой 3 & $\begin{array}{c}\text { Толщина, нм } \\
\mathrm{Bi}_{2} \mathrm{Te}_{3}, \%\end{array}$ & $\begin{array}{l}31 \\
8.4\end{array}$ & $\begin{array}{c}62 \\
8\end{array}$ \\
\hline
\end{tabular}

при использовании указанной модели величина MSE получалась большей. В этом случае для нанокомпозитов с номинальной концентраций наночастиц $\mathrm{Bi}_{2} \mathrm{Te}_{3} 60$ и 70\% наиболее подходящей оказалась модель с тремя слоями, что связано с возрастанием неоднородности из-за роста концентрации наночастиц. Величины MSE c учетом вышеизложенного приведены в табл. 2.

Полученные величины MSE свидетельствуют о хорошем соответствии построенной модели с экспериментальными данными. Экспериментальные (сплошные линии) и расчетные (пунктирные кривые) $\Psi$ и $\Delta$ для нанокомпозита концентраций наночастиц $\mathrm{Bi}_{2} \mathrm{Te}_{3} \quad 30 \%$ показаны на рис. 4. Из рис. 4 видно хорошее согласие между экспериментальными и расчетными (пунктирные кривые) данными.

Вычисленные в приближении Бруггемана концентрации и толщины слоев приведены в табл. 3 (для концентраций наночастиц $5,10,30,40,50 \%$ ) и в табл. 4 (для концентраций наночастиц 60 и 70\%).
Из представленных табл. 3 и 4 хорошо видно, что номинальные концентрации не соответствуют измеренным в ходе эллипсометрии. Это связано с кластеризацией в образцах и недостающая часть нанокристаллитов скапливается в кластерах, что будет показано далее. В работе [6] описана методика исследования корреляции между деполяризацией D и зернистостью и определения среднего размера зерен. Применительно к нашему случаю, эти исследования помогли оценить средние размеры микрокластеров, образованных группами наночастиц. Как видно из рис. 5, с увеличением энергии фотона (уменьшением длины волны) деполяризация образца сначала следует критерию Рэлея для шероховатой поверхности (т. е. для меньшего угла падения она больше, чем для большего). Однако при достижении энергии, соответствующей длинам волн 200-250 нм, наблюдается прямо противоположное поведение (т.е. деполя-

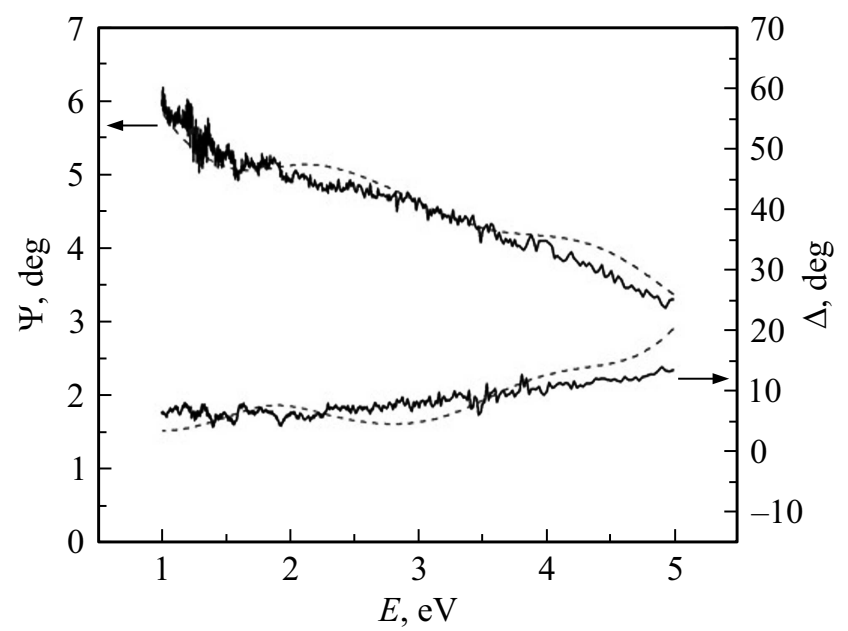

Рис. 4. Параметры спектроскопической эллипсометрии для нанокомпозита с концентрацией наночастиц $\mathrm{Bi}_{2} \mathrm{Te}_{3} \quad 30 \%$. Сплошной линией обозначены экспериментальные данные, штриховой — обозначены расчетные данные.

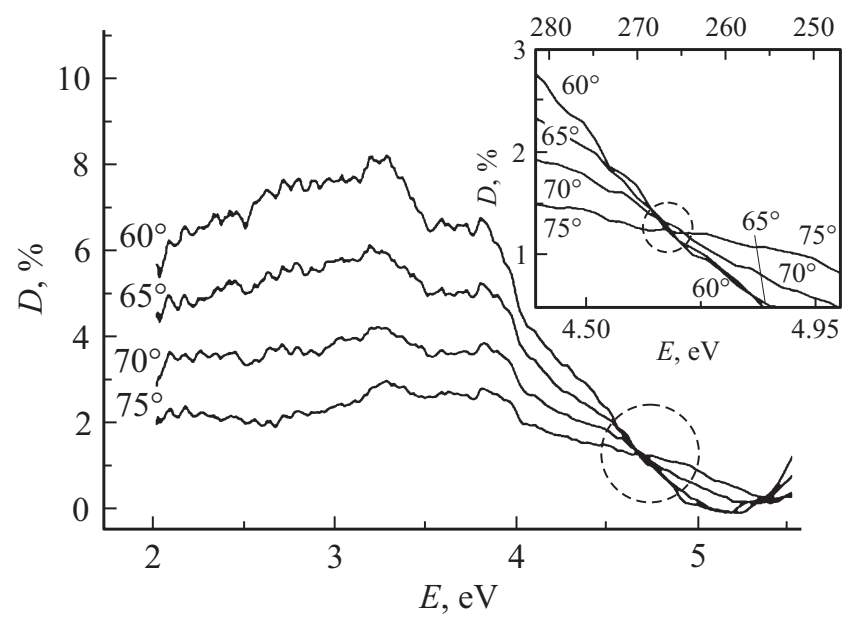

Pис. 5. Деполяризация D для нанокомпозита с $30 \%$ содержанием наночастиц $\mathrm{Bi}_{2} \mathrm{Te}_{3}$. 


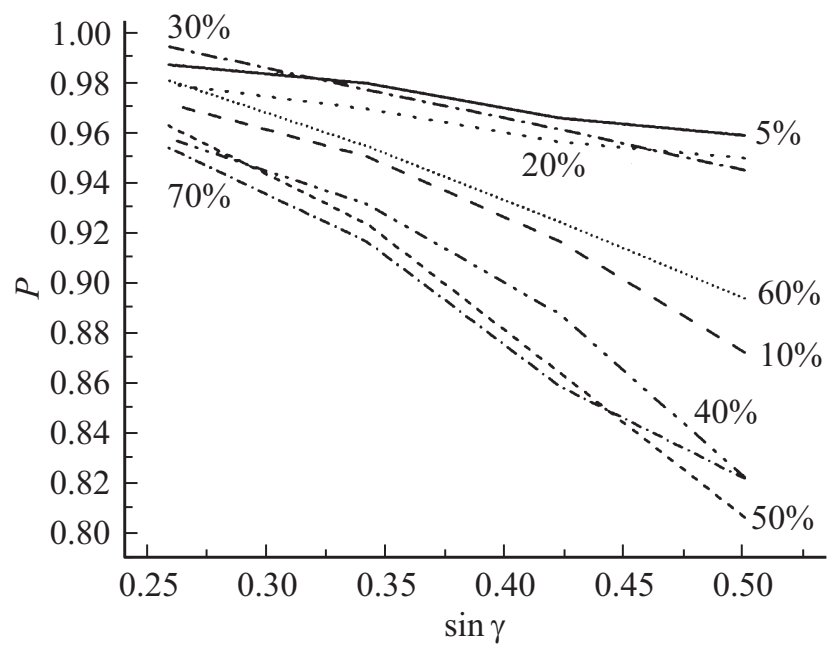

Рис. 6. Зависимость поляризации $P$ от синуса угла скольжения $\gamma$ при энергии фотонов 4 эВ (310 нм) для образцов разного состава.

ризация для меньшего угла падения меньше, чем для большего), как ясно видно на вставке к рис. 5. Согласно [6], значение 200-250 нм соответствуют средним размерам микрокластеров, образованных наночастицами теллурида висмута. Принимая во внимание, что средний размер наночастиц составляет 50 нм, можно заключить, что наночастицы в исследованном образце образуют микрокластеры, состоящие из 4-5 наночастиц. Следует отметить, что размеры кристаллитов, измеренных методом рентгеновской дифракции, относятся к одиночным кристаллитам, а не к кластерам.

На рис. 6 показана зависимость поляризации $P=(1-D)$ от синуса угла скольжения $\gamma$ (сумма углов падения и скольжения составляет 90). Как видно из рис. 6, поляризация $P$ уменьшается с увеличением угла скольжения. Иными словами, для образцов всех составов поляризация следует критерию Рэлея для шероховатых поверхностей со средним размером неоднородностей, меньшим длины волны, что для энергии фотона 4 эВ составляет $310 \mathrm{Hм}$.

Следует отметить, что эллипсометрические исследования проводились с использованием фокусирующего устройства и размер светового пятна на образце не превышал 250 мкм. Это позволило выбрать достаточно гладкие участки поверхности с наименьшей деполяризацией, а также правильно выбрать угол падения, при которых между эллипсометрическими параметрами и диэлектрической функцией имеется однозначное соответствие. При деполяризации, превышающей 5\%, обеспечение такого соответствия проблематично.

Поскольку наиболее предпочтительными (с точки зрения чувствительности) являются эллипсометрические измерения при углах, близких к углу Брюстера материала, то следовало бы восстанавливать диэлектрическую функцию по эллипсометрическим данным, полученным на углах, близких к $60^{\circ}$ (угол Брюстера полиэтилена при $n=1.5)$. Однако, учитывая реальную деполяризацию (рис. 5), только образцы с содержанием 5, 20 и 30\% $\mathrm{Bi}_{2} \mathrm{Te}_{3}$ одновременно удовлетворяют как критерию по деполяризации $(<5 \%)$, так и условию по углу $\left(60^{\circ}\right)$. Для остальных составов при восстановлении диэлектрической функции за основу брались эллипсометрические данные, полученные при углах 70-72॰ при которых деполяризация находилась в пределах допустимого уровня (рис. 6).

\section{4. Заключение}

Таким образом, нами получены и охарактеризованы методами дифракции рентгеновских лучей и спектров комбинационного рассеяния света нанокомпозиты матрицы LDPE с наполнителем в виде нанокристаллитов $\mathrm{Bi}_{2} \mathrm{Te}_{3}$.

Методами спектроскопической эллипсометрии обнаружено отклонение номинальных концентраций наночастиц $\mathrm{Bi}_{2} \mathrm{Te}_{3}$ от их реального содержания в нанокомпозите. Вследствие неоднородности нанокомпозитов предложены различные многослойные модели. Проведены вычисления на основе аппроксимации эффективной среды (Effective Medium Approximation, EMA) приближением Бруггемана, получена дисперсия мнимой и действительной частей диэлектрической функции. В данной работе объяснена природа неоднородностей в нанокомпозитах путем изучения деполяризующих свойств нанокомпозитов.

Работа выполнена при финансовой поддержке Фонда развития науки при президенте Азербайджанской Республики (гранты № EIF-BGM-3-BRFTF-2+/2017-15/02/1 и № EİF/MQM/Elm-Tehsil-1-2016-1(26)-71/16/1).

\section{Список литературы}

[1] L.D. Hicks, M.S. Dresselhaus. Phys. Rev. B, 47, 12727 (1993).

[2] Э.М. Годжаев, С.Ш. Кахраманов, К.Дж. Гюльмамедов, А.Ю. Гамзаева. Термоэлектричество, № 3, 21 (2013).

[3] W. Richter, H. Kohler, C.R. Becker. Phys. Status Solidi B, 84, 619 (1977).

[4] H. Fujiwara. Spectroscopic Ellipsometry: Principles and Applications (John Wiley \& Sons Ltd, 2007).

[5] D. Stroud. Superlat. Microstr., 23, 567 (1998).

[6] N. Mamedov, Y. Shim, H. Toyota, K. Wakita, N. Yamamoto, S. Iida. Phys. Status Solidi A, 203, 2873 (2006).

Редактор Г.А. Оганесян 


\title{
Optical properties of polyethylene filled with $\mathrm{Bi}_{2} \mathrm{Te}_{3}$ nanocrystallites
}

\author{
A.Yu. Gamzayeva', E.G. Alizade' ${ }^{2}$ N.T. Mamedov², \\ N.A. Abdullayev', I.R. Amiraslanov', Y.N. Aliyeva2, \\ Kh.N. Akhmedova ${ }^{2}$, G.H. Azhdarov', \\ K.Sh. Kahramanov', S.A. Nemov ${ }^{3}$ \\ ${ }^{1}$ Ganja State University, \\ Az-2003, Ganja, Azerbaijan \\ ${ }^{2}$ Institute of Physics, \\ Azerbaijan National Academy of Sciences, \\ Az-1143 Baku, Azerbaijan \\ ${ }^{3}$ Peter the Great St. Petersburg Polytechnic University, \\ 195251 St. Petersburg, Russia
}

\begin{abstract}
Composite mixtures of the low density polyethylene (LDPE) and powdered $\mathrm{Bi}_{2} \mathrm{Te}_{3}$ nanocrystals with different concentration of the constituents were prepared by thermal pressing. The obtained mixtures were preliminary characterized by $X$-ray diffraction and Raman scattering and then studied by spectroscopic ellipsometry over the photon energy range $1-6 \mathrm{eV}$. Using Bruggeman effective medium approximation and the values of optical constants obtained for polyethylene and $\mathrm{Bi}_{2} \mathrm{Te}_{3}$ crystal from spectroscopic ellipsometric measurements, the dielectric function of the ideally homogeneous mixtures of the considered constituents was calculated for compositions with 5, 10, 30, 40, 50, 60 and 70\% weight concentrations of $\mathrm{Bi}_{2} \mathrm{Te}_{3}$ nanocrystallites. The analysis of the depolarization peculiarities of the reflected light allowed to disclose samples' inhomogeneity caused by the clasterization of nanocrystallites with increasing weigh concentration of $\mathrm{Bi}_{2} \mathrm{Te}_{3}$ in polyethylene and to propose a most reliable optical model of the obtained composites.
\end{abstract}

